\title{
Le riverain, le citoyen et l'habitant : trois figures de la participation dans la turbulence éolienne
}

\author{
Sophie Le Floch \\ Géographe, CEMAGREF, 33612 Cestas cedex, France
}

\begin{abstract}
À un moment où la participation du public au débat sur l'aménagement du territoire est institutionnalisée, cet article tente de saisir les figures d'expression de la société civile dans un cas où la controverse règne, celui de l'énergie éolienne. S'intéresser au "public », terme si mal défini, suppose que l'on sorte du dilemme où il serait soit porteur d'intérêts particuliers, et en cela immanquablement disqualifié, soit préoccupé du seul intérêt général. Riverain, citoyen ou habitant, autant de figures d'une participation en quête de légitimité à partir d'une proximité géographique avec un projet en cours, d'un droit revendiqué à pouvoir débattre des enjeux locaux ou nationaux, ou bien d'une relation existentielle du fait de l'appartenance à un lieu. NSS continue d'explorer à traves cet article les multiples facettes d'une démocratie participative toujours incertaine.
\end{abstract}

La Rédaction

\begin{abstract}
Mots-clés : participation; habitant ; développement éolien ; géographie ; Finistère

Résumé - La participation, aujourd'hui consensuelle, fait l'objet d'une abondante littérature scientifique. Celle-ci est toutefois peu diserte sur les catégories du «public » qui en sous-tendent les différentes modalités. L'article examine les figures des « participants » et les espaces qui leur sont associés du point de vue d'acteurs impliqués dans le développement éolien : le riverain, décliné diversement ; le citoyen, peu mobilisé ; l'habitant, en émergence. Il conclut sur les risques et les promesses liés à la figure de l'habitant, susceptible d'osciller entre droit à être laissé tranquille et droit à participer.
\end{abstract}

\section{Keywords:} participation; local inhabitant; wind energy development; geography; Finistère

\begin{abstract}
Neighbour, citizen and local inhabitant: three characters engaged in wind turbine turbulence. Participation has become a consensual objective. It has been inscribed in different international and national regulatory texts - Rio Summit, Aarhus Convention, French 1995 Environmental Law - and generates a mass of academic work. Nonetheless, existing scientific literature deals little with the categories of "public" involved in the different modes of participation. This paper is based upon the assertion that improved participation requires greater in-depth definition of the targeted public. On the basis of empirical work dealing with social contestation about windfarms, the paper examines the different participating characters and their related spaces as perceived by public and private actors engaged in the development of wind energy production: the diversely defined neighbour - the neighbour as owner, the "oversensitive" neighbour; the (rarely mentioned) citizen; and the emerging local inhabitant. The promises and dangers of this emerging character are discussed: could there be a place in participation for the local inhabitant as a geographical human being - one whose experience of the environment matters, conceived as including both socio-political and sensitive dimensions -, in the current context where primacy is given to private spaces and where a new model of citizenship is created, based no longer on associative forms of interaction but on the right to be left alone and on the right to exclude (Mitchell, 2005)?
\end{abstract}

Il semble qu'il y ait aujourd'hui un consensus autour de l'objectif de la participation. Celle-ci, portée par la préoccupation environnementale, est désormais inscrite dans différents textes internationaux - sommet de Rio,

Auteur correspondant : sophie.le-floch@cemagref.fr convention d'Aarhus... En France, dans un contexte de changements des processus décisionnels à l'œuvre depuis les années 1970, elle est progressivement institutionnalisée à partir de la décennie 1990 (Mermet et BerlanDarqué, 2009). Cependant, sa mise en pratique soulève bien des questions. Une abondante littérature scientifique souligne ainsi les flous conceptuels inhérents à la notion 
telle qu'elle est mobilisée par les acteurs de l'aménagement du territoire, en vue d'améliorer sa mise en œuvre : formulations d'objectifs démocratiques, mise en place de processus ad hoc, etc. (Blondiaux et Sintomer, 2002). Elle s'appuie notamment sur des typologies reflétant divers degrés d'intensité et de réciprocité des échanges entre parties prenantes (Arnstein, 1969 ; Beuret et al., 2006 ; Blondiaux, 2004).

Les conceptions du «public » associées à différentes conceptions ou modalités de la participation apparaissent dans ces travaux, mais elles font rarement l'objet d'analyses spécifiques. Globalement, il ressort que les figures du public concerné tournent principalement autour d'une opposition que la tradition française consultative, fondée notamment sur le dispositif de l'enquête publique, a largement contribué à asseoir : l'opposition «riverain/citoyen». Le premier serait porteur d'intérêts particuliers ; sa figure est exacerbée et disqualifiée par les lectures NIMBYistes ${ }^{1}$ émanant d'aménageurs ou de scientifiques (Jobert, 1998). Le second serait " amateur de l'intérêt général et de sa mise en discussion démocratique »(Fourniau, 2008). Avec l'invention du débat public, qui donne naissance au « citoyen en tant que riverain », on aurait pu s'attendre à l'estompement de cette distinction. Celle-ci réapparaît pourtant à l'occasion de diverses expérimentations - conventions de citoyens, par exemple - sous la forme « citoyen ordinaire / citoyen concerné ». Le premier, le « profane » qui consent à débattre, serait préoccupé du seul intérêt général ; le second, concerné territorialement, serait mobilisé car partie prenante et s'apparenterait donc au riverain (ibid.).

Au-delà des réflexions sur le couple «riverain/ citoyen », la littérature apporte quelques éclairages sur d'autres catégories du public. Claeys-Mekdade (2001) recense treize termes plus ou moins explicitement mobilisés par les acteurs de démarches participatives - «population », « habitants ", « usagers "... À l'aide du dictionnaire, elle en propose une définition usuelle avant de mentionner que, dans la pratique, cette catégorisation ne résiste ni aux hybridations ni aux cohabitations contradictoires. Neveu (2003), examinant les processus de catégorisation institutionnelle à l'œuvre dans le cadre de la politique des quartiers, distingue l'« Habitant » des « habitants »: le premier terme ferait référence à un acteur collectif né des luttes urbaines des années 1960 et 1970 ; le second correspondrait à une vision moins nette des habitants. Or, cette figure floue et changeante est permanente dans les discours et les pratiques de la participation, qu'il s'agisse de la politique de la ville, des grands projets d'infrastructures, etc.

\footnotetext{
1 NIMBY : Not in my back yard (« pas dans ma cour »).
}

De cette lecture des analyses conduites dans ce domaine, il ressort donc que les travaux portant attention au public - ses catégories, ses statuts... - mériteraient d'être prolongés. En effet, une meilleure mise en œuvre de la participation passe sans doute aussi par une réflexion sur la façon de concevoir le public cible de la part de tous ceux qui sont amenés à être impliqués dans les démarches participatives (Carrel, 2007 ; Neveu, 2003). Or, les enjeux principaux pour clarifier la conception du public se posent notamment en termes de spatialisation et d'organisation des habitants auxquels un certain pouvoir pourra être attribué. Il semble donc qu'il y ait une place pour une réflexion au carrefour de la géographie et des sciences politiques. Sur la base de travaux empiriques visant à mieux comprendre les contestations sociales autour de l'implantation d'éoliennes ${ }^{2}$, nous examinerons les questions suivantes: Dans les discours relatifs aux démarches de participation prônées et/ou mises en œuvre, quelles figures des « participants » esquissent les acteurs impliqués dans le développement éolien et quels espaces ces figures mobilisent-elles selon eux ? Il ne s'agit pas d'analyser les jeux d'acteurs ni les évolutions de la pensée du collectif dans un contexte de reconfiguration rapide de ce dernier ${ }^{3}$. Il ne s'agit pas non plus de caractériser les représentations sociales des éoliennes ni les processus conduisant à la cristallisation de conflits autour de ces équipements. Le propos porte sur la façon dont se modifie la vision qu' ont du « public » et de ses différentes catégories - « habitant », « citoyen »...-des acteurs ayant voix dans le débat social et/ou l'action en matière d'aménagement et de devenir des territoires, au-delà de la seule problématique éolienne; et ce, alors que ce "public » s'impose peu à peu comme acteur ordinaire dans des structurations politiques elles-mêmes changeantes, en y important ses spatialités propres, ses goûts esthétiques, etc. (Charles et Kalaora, 2003).

La perspective adoptée est celle d'une approche de géographie croisant un questionnement politique ${ }^{4}$. Cette approche postule que l'être humain est un être géographique, c'est-à-dire un être social dont l'existence nourrit la matérialité et l'étendue du monde, de même qu'elle s'en nourrit. En tant qu'êtres de chair, de sens,

\footnotetext{
2 Le propos développé ici s'appuie sur une recherche intitulée "Le développement durable à l'épreuve des paysages d'Éole », réalisée en partenariat avec Marie-José Fortin, de l'Université du Québec à Rimouski, dans le cadre du programme Paysages et développement durable financé par le MEEDDM (2005-2008).

3 Ailleurs, nous proposons une lecture critique des processus participatifs pensés et mis en œuvre à l'occasion du développement éolien (Le Floch et Fortin, à paraître).

4 Bussi (2001) rappelle que, traditionnellement, la géographie (française) est peu soucieuse des questions de démocratie et de participation.
} 
d'intentionnalité, nous incorporons dans nos actions cette matérialité et cette étendue, tout comme les transformations de ces dernières sont marquées par nos actions (Relph, 1976 ; Berque, 2000). À travers l'analyse de discours sur les pratiques, 1 'objectif est de comprendre simultanément les significations attribuées à des objets, à des environnements, aux changements qui les affectent, ainsi qu'aux groupes sociaux en présence et à leurs relations. Il s'agit donc de « saisir les arts de faire spatiaux d'une entité sociétale complexe, l'individu » (Lussault, 2000), en mobilisant une conception de l'espace non pas comme arène où " ont lieu » les relations sociales, mais comme propriété de ces relations (Massey, 1999 ; Corsin Jimenez, 2003). Le dire et le faire sont conçus comme entretenant des relations d'étroite proximité. « Tout dire est un faire social » : qualifier un lieu, justifier une action, nommer un espace... Chaque acteur d'une situation produit et diffuse des énoncés qui, autant que les résultats matériels de l'acte, permettent « d'appréhender les logiques et le sens de l'action » (Lussault, 2000).

Notre analyse s'appuie sur un matériau constitué d'entretiens peu directifs, réalisés entre mai 2006 et juin 2008 auprès de soixante acteurs impliqués dans les débats autour du développement éolien dans le Finistère ${ }^{5}$. Les champs d'intervention des enquêtés sont divers: environnement, urbanisme, industrie, développement local, paysage. Leur périmètre d'intervention est également varié : du site éolien à la région, en passant par la commune, l'intercommunalité, le département. Les enquêtés comprennent des représentants de: douze collectivités territoriales (conseil régional de Bretagne, conseil général du Finistère, communautés de communes...); cinq services déconcentrés de l'État, dont la Direction régionale de l'industrie et de la recherche (DRIRE), la Direction régionale de l'environnement (DIREN), la Direction départementale de l'équipement (DDE) du Finistère; dix-huit bureaux d'études (développement éolien, paysage, médiation environnementale); six associations (environnementalistes, riverains...) ; s'y ajoutent deux porteurs de projet et quelques habitants. Ces personnes ont été invitées à témoigner de leur expérience relative à l'ensemble des projets éoliens dans lesquels elles ont été impliquées. Leurs récits s'appuient sur de multiples exemples de projets d'implantation d'éoliennes, ainsi que sur des expériences d'élaboration d'outils d'aménagement et de planification plus généraux (chartes départementales, schémas locaux de développement éolien notamment).

\footnotetext{
5 Au moment de la recherche, la dynamique éolienne, après avoir débuté en Languedoc-Roussillon, touche particulièrement ce département, qui est le premier au niveau national à se doter d'une charte des éoliennes, en 2002. En 2006, le Finistère compte 13 parcs, comptabilisant 66 turbines pour un total de 68 MW raccordés au réseau (source : DDE du Finistère).
}

Nous exposerons successivement les différentes figures du public que font apparaître en filigrane les acteurs interrogés : d'abord la figure du riverain, qui se décline diversement; puis celle du citoyen, très peu mobilisée; enfin, celle de l'habitant, qui semble en émergence. Auparavant, nous rappellerons le contexte d'émergence de la participation dans la législation française ainsi que dans les débats et démarches autour des projets d'implantation d'éoliennes.

\section{La participation devenue évidence}

Historiquement, en France, l'enquête publique est instaurée en 1810 dans le souci de défendre les droits de certains propriétaires, riverains de projets d'équipement ou d'aménagement. Mais il faut attendre la décennie 1970 et la montée de la préoccupation environnementale pour que le thème de la participation apparaisse.

Ce thème est $\mathrm{d}$ 'abord décliné sous la forme du droit à l'information du public quant aux conséquences environnementales de certains projets, en particulier dans la loi de 1976 relative à la protection de la nature, qui crée l'étude d'impact. Puis, la consultation préalable des citoyens en matière d'urbanisme devient obligatoire dans le contexte de la décentralisation au début des années 1980. La loi Bouchardeau de 1983, relative à la démocratisation de l'enquête publique et à la protection de l'environnement, n'a pourtant pas pour objet la participation en tant que telle, mais l'information, à la fois du public - sur le projet - et de l'administration - sur d'éventuelles réactions au projet. Les années 1990 marquent un tournant. À la suite des circulaires Bianco (1992) et Billardon (1993) prévoyant la concertation en amont de la conception de certains grands projets, la loi Barnier de 1995 relative à la protection de l'environnement reconnaît le principe de participation et crée la Commission nationale du débat public (CNDP). Cette évolution fait écho à la reconnaissance de la participation au niveau international, notamment via la convention d'Aarhus, adoptée en 1998 (mais entrée en vigueur en France en 2002) en application du principe 10 de la Déclaration de Rio ${ }^{6}$. Depuis quinze ans, maints textes étendent le principe de la participation à de multiples domaines : loi nationale de 2002 relative à la démocratie de proximité, rendant les conseils de quartier obligatoires dans les villes de plus de 80000 habitants ; charte française de l'environnement de 2004 ; Convention européenne du paysage de 2000 (entrée en vigueur en France en 2006)...

\footnotetext{
6 L'idée de base est que la "meilleure façon de traiter les questions d'environnement est d'assurer la participation de tous les citoyens concernés » (http://www.un.org/french/ events/rio92/aconf15126vol1f.htm).
} 
Paradoxalement, au vu d'un tel contexte, le développement éolien, qui suppose l'implantation d'objets sans commune mesure avec les constructions ou équipements existants ${ }^{7}$, est à l'origine confié presque exclusivement aux acteurs privés, avant l'élaboration d'outils de régulation. Cette vision décentralisée rompt avec la vision jusque-là dominante en France, notamment en matière d'aménagement du territoire. À l'époque, les procédures du permis de construire et de l'étude d'impact pour les projets de plus de 12 millions de francs demeurent les seuls outils réglementaires. Il faut dire qu'en France, la politique environnementale élaborée dans le cadre de la diplomatie climatique internationale ${ }^{8}$ est traduite en une politique énergétique, essentiellement quantitative et mobilisant des outils financiers ${ }^{9}$. Si le respect de l'environnement et l'avis des collectivités territoriales sont mentionnés comme conditions à remplir, le souci de la dissémination géographique prime pour des raisons énergétiques : absorption de l'électricité par le réseau existant... En $2003^{10}$, d'autres dispositifs réglementaires deviennent obligatoires : le permis de construire pour les équipements éoliens de plus de 12 mètres de haut, l'étude d'impact pour des sites dont la puissance excède 2,5 MW et l'enquête publique. Mais ce n'est qu'en juillet 2005 que le premier dispositif réglementaire spécifique voit le jour : la zone de développement éolien ${ }^{11}$. S'il s'agit d'un outil énergétique plus qu'urbanistique, son volet « environnement $^{12}$ » ouvre à la possibilité de projets de paysage au niveau des territoires.

Dans l'intervalle, la question environnementale, interprétée notamment en termes de «paysage » (Le Floch et

\footnotetext{
7 Une éolienne peut atteindre 150 mètres en bout de pale, tandis qu'un clocher d'église avoisine plutôt les 30 mètres et un pylône à très haute tension les 54 mètres. Cette mise en perspective d'échelles est d'ailleurs souvent reprise par les opposants aux éoliennes.

8 Le protocole de Kyoto, signé en 1997, marque une étape essentielle de la Convention-cadre des Nations unies sur les changements climatiques. Il contraint 36 pays industrialisés à réduire leurs émissions de gaz à effet de serre (GES) d'au moins $5 \%$ par rapport à leur niveau de 1990 d'ici 2008-1912. Les pays émergents (Chine, Inde, Brésil, notamment) ont une obligation d'inventaire.

9 La France prend les premières mesures de réduction des émissions de GES en 1990 et commence à s'intéresser à l'éolien à partir de 1996. Deux régimes de soutien se succèdent: le régime d'appel d'offres (programme Éole 2005, lancé en 1996) ne donnant pas les résultats escomptés (11 MW raccordés fin 1999, pour un objectif de 250 à 500 en 2005), un régime de tarifs de rachat garantis prend le relai à partir de 2000 (fin 2006, lors de nos enquêtes, $1635 \mathrm{MW}$ sont raccordés, selon l'ADEME).

${ }^{10}$ Loi n $^{\circ}$ 2003-8 du 3 janvier 2003 relative aux marchés du gaz et de l'électricité et au service public de l'énergie.

${ }^{11}$ Créée par la loi de programmen ${ }^{\circ}$ 2005-781 du 13 juillet 2005 fixant les orientations de la politique énergétique.
}

Fortin, 2008), va resurgir à l'initiative des acteurs locaux, publics comme privés. En particulier, les services instructeurs des demandes de permis de construire (préfecture, DDE), ainsi que les opérateurs éoliens privés, se sentent mal à l'aise face aux questions de plus en plus vives d'élus, d'habitants, voire d'opposants organisés. Dès le début des années 2000, des conflits éclatent, conduits souvent par des opposants qui n'envisagent pas d'autres solutions que le retrait des projets ${ }^{13}$. Face à ce qu'ils perçoivent comme une défaillance des politiques publiques, les acteurs locaux engagent des processus de concertation à l'échelle de territoires: charte départementale des éoliennes du Finistère parue en $2002^{14}$; schémas locaux de développement éolien, dont le premier est adopté en juillet $2004^{15}$; schéma régional adopté en $2006^{16} \ldots$. Dans la conduite de ces dialogues territoriaux, le souci du public est constant. Si la charte souligne, entre autres, la nécessité pour les opérateurs d'informer les associations et les riverains et de dialoguer avec eux, le schéma régional - sous-titré Du partage de connaissances à l'accord collectif - va jusqu'à développer un guide de la concertation. Au nom de l'exigence de qualité qui émanerait des citoyens, il semble vouloir doter la puissance publique d'un rôle de "régulation publique et démocratique". Alors que les premiers outils spécifiques apparaissent tout juste et que la seule injonction de participation est apportée par l'obligation de l'enquête publique, tout se passe comme si les représentants et les services de l'État, ainsi que les opérateurs privés, impulsaient une technicisation de l'enjeu de participation: faire de celle-ci un élément du montage des projets au même titre que le potentiel éolien ou le raccordement au réseau.

Les acteurs du développement éolien enquêtés confirment cette préoccupation $\mathrm{du}$ " public ». L'analyse des entretiens montre cependant que leur vision de ce public est loin d'être claire.

\section{Trois figures pour une « matière molle »}

Un double paradoxe apparaît. D'un côté, on considère que «les gens» n'ont pas d'avis sur la question de

\footnotetext{
12 Ce volet comprend des éléments relatifs à la protection des paysages, des monuments historiques et des sites remarquables et protégés.

${ }^{13}$ En 2004, 25\% des 175 permis de construire délivrés faisaient l'objet d'un recours auprès d'un tribunal administratif.

14 http://www.finistere.equipement.gouv.fr/IMG/pdf/ Charteoliennes_cle584757.pdf

15 http:/ / www.cc-chateaulin-porzay.fr/ledeveloppement eolien_.php

16 http:/ / www.finistere.equipement.gouv.fr/IMG/pdf/ Guide_eolien_BZH_cle1be9f1.pdf
} 
l'énergie : il serait donc possible d'informer, c'est-à-dire, étymologiquement, de "donner une forme à » cette "matière molle ». D'un autre côté, on craint que des réactions négatives ne surgissent çà et là et n'entravent le bon déroulement de l'élaboration des projets, en ces temps où la participation est un sujet sensible : le recours à des dispositifs participatifs permettrait de ménager les susceptibilités.

«Au départ, la population n'a pas forcément un... Les dés ne sont pas jetés dès le départ, quoi... C'est qu'il y a une espèce de matière molle, là, et $c^{\prime}$ est un peu la manière dont ça va se dérouler, que cette matière va être plutôt favorable à l'émergence d'un projet ou défavorable. [...] La problématique énergétique est loin de leurs préoccupations.» (Médiateur, $\mathrm{BE}^{17}$ en sciences humaines.)

Ainsi, le public est globalement perçu comme un réceptacle passif d'où seraient susceptibles d'émerger quelques individus réagissants. Les acteurs du développement éolien distinguent cependant quelques figures, dans leur effort de saisir la complexité de ce qui leur apparaît de manière insatisfaisante comme une masse indifférenciée $^{18}$

\section{Le riverain}

\section{Une dimension centrale : la distance/proximité}

Dans les discussions autour de l'implantation d'éoliennes, la notion de riverain est d'emblée centrale. Elle désigne celui qui est quotidiennement à proximité d'éoliennes et qui, simplement de par cette position spatiale, est susceptible de réagir à leur présence. Au cœur de cette définition, il y a donc une question de distance entre la maison du riverain et les éoliennes. Elle est toutefois indissociable d'une dimension politique, même si celle-ci est conçue négativement et tend à être évincée: le riverain est celui duquel il faut écarter l'éolienne afin de minimiser les risques de contestations. En quelque sorte, c'est une figure construite de manière à être mise à l'écart.

«Le développeur, quand il cherche un site, il cherche un site venté où il y a de la place. Donc, la première chose qu'il va faire, $c^{\prime}$ est $d^{\prime}$ acheter une carte des vents [...] puis après, ce qu'il fait, c'est qu'il prend son calque, Photoshop, ou n'importe quoi, et puis il fait des cercles de 500 mètres autour de toutes les maisons. » (Paysagiste, BE en développement éolien.)

\footnotetext{
17 BE : bureau d'études.

18 Un spécialiste en médiation environnementale avoue sa difficulté à saisir, en particulier, le ou les profils de l'opposant éolien : "Je veux pas rentrer dans ce truc-là. Je pense que la sociologie de l'opposant, on n'a pas réussi à la déterminer précisément. »
}

$\mathrm{Au}$ début du développement éolien, aucune norme n'existe relativement à la distance aux habitations. C'est donc empiriquement que les acteurs locaux en construisent. Ils s'appuient sur le volet acoustique de l'étude d'impact - mesure du niveau d'émergence sonore -, qui doit déterminer cette distance en conformité avec la loi «bruit » de 1992. Sur cette base, la distance moyenne recommandée par l'ADEME est de 400 mètres. Dans la pratique, si au départ il est question de 350400 mètres, les acteurs finistériens s'accordent pour retenir 500 mètres. La distance s'accentue encore à l'initiative des développeurs, alors que les revendications des associations d'opposants se font plus précises - en particulier à la suite des recommandations de l'Académie de médecine en 2006. Cette même année, un responsable de bureau d'études confirme que la distance retenue est désormais de 1000 mètres. Les raisons de cette augmentation sont floues; elles ne se limitent pas, en tout cas, au seul bruit. Le postulat semble être que l'intensité de la réaction aux éoliennes est une fonction linéaire de la distance.

«Plus on se rapproche de l'habitat, plus les positions... enfin, le riverain proche aura une position affirmée par rapport à ce parc-là.» (Médiateur, BE en médiation environnementale.)

«Y avait pas de normes. $Y$ en a toujours pas d'ailleurs, de très précises en France, bien que maintenant on évoque souvent 350 mètres, 400 mètres. Nous, on a préféré prendre plus de marge. On a dit que 500, c'était pas mal. » (Secrétaire de mairie.)

La figure du riverain, très présente dès les premiers temps des débats autour de l'éolien, se décline différemment.

\section{Le riverain propriétaire : une définition marchande}

Parmi les promoteurs et les opposants - les acteurs les plus " militants ", donc -, la figure du riverain propriétaire s'impose, qu'ils la mobilisent simplement ou qu'ils parlent en son nom. Elle correspond à une logique marchande. Le propriétaire est celui avec qui les promoteurs négocient, moyennant rétribution, l'implantation d'éoliennes. Le montant des revenus alloués est souvent négocié secrètement; il oscillerait entre 4000 et 7500 euros par éolienne et par an.

«Donc y a cinq autres agriculteurs avec nous [qui ont des éoliennes sur leurs terres]. Ils touchent 7500 euros par an et par éolienne [...]. Donc c'est moi qui fais les prix, hein ! [...] Mais c'est énorme... c'est-à-dire que, dans certains cas, c'est plus que le revenu du troupeau laitier, quoi... [...]Parce que, à ce prix-là, ils ont rien à faire, si vous voulez... » (Porteur de projet.)

«On a des voisins qui trouvent ça affreux [les éoliennes] [...]. Mais ces gens-là ont chez eux des pylônes de haute tension et qui pendant la nuit, quand il y a du brouillard, font 
du bruit du tonnerre aussi, et ça, ça les dérange pas. [...] Parce que ces gens-là ont été indemnisés pour les poteaux qu'ils ont eus, hein ! [...] Je pense que ça jalouse un petit peu, parce que, finalement, le fait d'avoir une éolienne chez soi, faut quand même dire les choses comme elles sont... c'est un peu comme si vous gagniez un petit peu au loto. » (Riverain accueillant une éolienne sur ses terres.)

Certains enquêtés pensent que la négociation sur le mode financier serait en passe de s'étendre. Selon un porteur de projet, l'expression d'une demande sociale pour une participation plus large serait motivée par la volonté de négocier des compensations financières. Un développeur affirme que celles-ci sont parfois versées par le porteur de projet : le silence de certains riverains serait acheté. Un paysagiste expose les pratiques d'un développeur avec lequel il travaille, qui tente d'organiser les indemnisations aux riverains grâce à ce qu'il nomme le «système de la zone d'intérêt ».

«Un peu bizarres, ces réunions publiques. [...] Les gens se disent: “Ah bon! Si on me demande mon avis, c'est que je vaux quelque chose... Donc, combien vous pouvez me donner pour que je me taise ? [...] Comment je vais monnayer mon avis ?" » (Porteur de projet.)

« La première chose qu'ils [les personnes du BE de développement] ont fait faire, c'est qu'ils ont été voir tous les propriétaires des zones : ils travaillent avec le système de la zone d'intérêt. [...] Et puis on leur dit : "Voila, on vous propose une promesse de bail que vous signez [...]. Et puis on vous proposera à terme un projet ; si vous avez signé et que vous n'avez pas une éolienne, au final, vous aurez quand même quelque chose." » (Paysagiste, BE.)

La marchandisation des rapports au riverain est jugée préjudiciable par certains développeurs dans un contexte de concurrence foncière entre promoteurs. Elle contribue à renforcer la figure du propriétaire riverain au détriment de celle du citoyen, ce qui pourrait conduire à la fabrication de «frustrés » se muant en opposants. De plus, une mauvaise image - «commerciale» - de l'opérateur finirait par circuler. Les promoteurs entretiennent donc des rapports paradoxaux avec la figure du propriétaire riverain : s'ils contribuent à la façonner et s'en servent, ils s'inquiètent aussi de l'image qu'elle renvoie d'eux.

"Il y avait une prospection foncière, une pression foncière extrêmement forte [...]. Finalement on avait des propriétaires frustrés qui se retiraient et qui, d'ailleurs, pouvaient se retrouver dans des associations d'opposants. [...] Il y a quand même une concurrence derrière qui un peu dégrade la qualité... l'image de l'opérateur. Les riverains peuvent très bien avoir une impression que cet opérateur... est ici uniquement à but commercial. » (Médiateur, BE en médiation environnementale.)

Lorsqu'ils critiquent les opposants, les acteurs du développement éolien dénoncent en eux cette figure marchande. L'opposant serait ce propriétaire insistant sur la dépréciation de son patrimoine du fait de la proximité d'éoliennes ; le riverain serait celui qui pense avoir acheté le paysage avec sa maison. Le phénomène NIMBY serait propre à cette figure du propriétaire riverain, éminemment égoïste et vénal, que l'on trouverait en particulier sur le bord de mer - là où la valeur du paysage serait la plus élevée.

"Ces gens-là vont estimer que lorsqu'on va envisager de mettre des éoliennes à 450 ou 500 mètres de leur habitation, ça va modifier de manière importante leur cadre de vie. D'autant plus que c'est souvent [...] une population de bord de mer qui [a] le sentiment en achetant [sa] maison ou en habitant dans [sa] maison, que le paysage [lui] appartient. » (Responsable d'études d'impacts, BE en développement éolien.)

\section{Le « riverain hypersensible » ou la primauté de l'intimité}

Une autre conception du riverain est à l'œuvre, largement présente chez tous ceux qui s'expriment sur le développement éolien, depuis les promoteurs de projets jusqu'aux opposants organisés, en passant par les acteurs publics : celle de l'individu soucieux de sa sécurité, en particulier sanitaire, et, plus largement, soucieux de l'intégrité de sa sphère privée.

Dans ce cas, le riverain est celui qui, de par sa proximité physique quotidienne avec les éoliennes, est susceptible de ressentir les effets de ces dernières « dans sa chair ». L'éolienne possède ce qui est vécu par tous ceux qui se sont trouvés à un moment ou à un autre à son pied comme une «violence matérielle» (Le Floch et Fortin, 2008): par ses caractéristiques physiques, elle vient rappeler, de manière exacerbée, combien la matérialité des choses compte dans notre expérience du monde. Tous les acteurs enquêtés, y compris les plus ardents défenseurs de l'énergie éolienne, admettent que cette violence matérielle puisse générer, pour ceux qui vivent à proximité, un sentiment d'insécurité, une peur. Une peur qui serait "irrationnelle», bien que souvent masquée par des arguments " rationnels », construits sur la base de perceptions : visuelles (argument du paysage) et sonores (argument du bruit).

«Les gens qui sont contre, généralement, bon, c'est surtout la peur des riverains. [...] Et c'est brutal, ces machines ! Donc, quelque part, y a une forme de rejet, d'inquiétude, c'est... c'est légitime, hein, quelque part. Par contre... ce qui est agaçant, $c^{\prime}$ est que les gens vont dire qu'ils sont pour la préservation des paysages, mais ils savent même pas ce que c'est que le paysage ! » (Paysagiste, conseil général.)

"[Des riverains s'opposent] [p]arce que, quand ils se lèvent, chez eux, le matin, ils les voient. On peut comprendre. Je les blâme pas pour autant, c'est un constat, point. [...] On habille le refus de la proximité par les arguments plus... économiques, rationnels, etc., qu'on trouve, hein !» (Maire.) 
Des responsables d'associations d'opposants, voire des acteurs publics, soulignent l'importance du ressenti des habitants à proximité des éoliennes. Ils rapportent des témoignages sur la façon dont la vie des personnes peut être affectée, tout en précisant que toutes n'ont pas la même sensibilité: stress, problèmes de sommeil, maladies de peau, etc. Ce qui serait insupportable à certains riverains, ce serait la présence - visuelle, sonore permanente de l'éolienne à l'intérieur même du foyer domestique, donc au plus profond de la sphère privée. Une présence, non voulue, qui serait susceptible de tourner à l'obsession; qui, finalement, serait vécue comme une atteinte à l'intégrité même de la personne, de la famille.

«Au fur et à mesure de l'implantation des éoliennes, on s'est aperçu que les riverains - ils ne sont jamais nombreux - subissaient des troubles divers. [...] Il y a la psychologie du bruit supporté ou supportable. Mais il y a aussi les effets du bruit sur l'organisme, quand même. Les bruits insidieux [...] qui agissent sur le corps. Sans que les gens en soient conscients. » (Responsable d'une association environnementaliste opposante.)

« Le point le plus délicat, en fait, au niveau du voisinage, c'est le bruit. C'est le bruit, parce que... en dehors de l'aspect psychologique du bruit, y a des trucs qui ne sont pas mesurés, comme les différences de gammes de fréquence ou les infrasons ou toutes ces choses-là... " (Chargé de mission énergies renouvelables, DIREN.)

«Ça vaut le coup de voir où il habite, parce que, quand vous serez dans son séjour, vous ne verrez que des éoliennes. D'un côté ou d'un autre. C'est fou ! Même assis sur ses toilettes! Ah! oui oui. Ah! mais c'est... envahissant. » (Membre d'une association environnementaliste opposante.)

\section{Le citoyen}

La figure du citoyen est relativement peu présente dans les entretiens analysés. Le terme est pratiquement absent, tout comme ceux de démocratie, de délégation de pouvoirs... auxquels il est censé être associé ${ }^{19}$. Pour l'essentiel, cette figure demeure « virtuelle».

\section{Une figure essentiellement virtuelle}

Le citoyen est la figure que tous les acteurs du développement éolien, «pro » ou « anti », appellent de leurs vœux: c'est l'individu capable de débattre des enjeux énergétiques à différentes échelles, de délibérer à propos des orientations collectives. De l'avis général, ce

\footnotetext{
${ }^{19}$ La citoyenneté est en effet définie en termes politiques dans le contexte de sociétés démocratiques (Painter et Philo, 1995).
}

citoyen n'existerait pas à l'heure actuelle, ou alors au stade embryonnaire ; au-delà du riverain, le public est perçu comme un être sans avis constitué ni contextualisé sur la question éolienne. Mais la façon dont les acteurs mettent l'accent sur les notions d'information et de communication - actions certes susceptibles de donner un certain pouvoir aux personnes et donc d'accroître leur capacité d'agir, au moins dans le cas de l'information (Beuret et al., 2006) - révèle plutôt leur désir de façonner les positions du public que d'en faire émerger des citoyens au sens plein du terme ${ }^{20}$.

\begin{abstract}
« La concertation est très axée là-dessus, sur la transparence, l'association d'un maximum de participants dès le départ pour faire en sorte que ce projet ne soit pas élaboré à côté des gens, mais avec les gens, même si... ils auront certainement peu de choses à dire, mais au moins qu'ils soient bien au courant... Évidemment, ils auront des choses à dire, mais... c'est surtout une phase où ils vont apprendre beaucoup de choses.» (Médiateur, BE en médiation environnementale.)
\end{abstract}

\section{Les opposants organisés, porte-parole de « citoyens handicapés »}

Les opposants organisés revendiquent finalement assez peu la participation de la population aux décisions. Certes, dans les discours qu'ils versent dans le débat social - interviews dans la presse, textes diffusés sur Internet... -, ils dénoncent souvent l'absence de concertation lors de l'élaboration des projets. Mais, dans les entretiens, ces aspects ne donnent pas lieu à d'amples développements. Plutôt, ils insistent longuement sur le fait qu'eux-mêmes doivent être entendus, notamment en tant que porte-parole de la minorité de riverains hypersensibles qu'ils voient comme des «citoyens handicapés »: selon ces opposants, ces derniers ne s'expriment dans aucune procédure participative, car ils seraient persuadés, d'une part, qu'ils ne possèdent pas de connaissances légitimes sur le sujet, d'autre part, qu'il n'y a pas de place pour les éléments purement subjectifs, émotionnels, dans le débat politique. Que ces personnes soient une minorité n'est pas, pour les opposants organisés, une raison valable de la non-prise en compte de leurs points de vue - ils sont bien conscients de ce que l'opposition se construit sur la base d'un petit nombre de gens. Un responsable d'association relate ainsi ses pressions auprès d'un préfet pour que celui-ci constate sur place l'expérience qui est celle de riverains stressés jusqu'à en être malades.

« Je lui dis : “Écoutez, M. le préfet, j’y suis allé un soir de tempête, tout fermé, à l'intérieur de la maison on entendait les éoliennes ! De dehors on ne les entendait pas !" Il m'a dit :

\footnotetext{
${ }^{20}$ Voir Le Floch et Fortin (à paraître).
} 
“Monsieur, c'est pas vrai. - Écoutez, M. le préfet, prenez votre voiture et allez-y, tout seul, un soir, vous verrez !" Et il y est allé. Réunion suivante : "Ah ! oui, M. [X], vous aviez raison. C'est curieux." »

Si les opposants organisés ont un souci de la démocratie, c'est celui d'une démocratie de proximité, où la légitimité de parole tient d'abord à la proximité spatiale avec l'objet dont on parle, même si cette parole est minoritaire. Les responsables d'un comité local de défense utilisent les termes de « démocratie locale ». Mais cette minorité entend être considérée non pas d'un point de vue strictement politique, mais selon une dimension plus subjective : les éléments liés à l'intégrité de la sphère privée - individu, famille, maison d'habitation reviennent. Cette figure s'apparenterait plus à celle du riverain qu'à celle du citoyen.

\footnotetext{
« D'un point de vue démocratie locale, je trouve ça vraiment très, très triste, quoi [de ne pas être entendu]. [...] Alors, après, on a sorti des arguments sur la sécurité, sur le bruit, sur... qu'est-ce qu'il y avait? la sécurité, le bruit, l'incendie, la pollution; bon, certains sont importants, d'autres moins. [...] Ici, quand tu es en bas du champ, on voit la maison et puis au-dessus les éoliennes. Donc, par exemple, si nous on veut vendre demain, on fait comment? [...] Ma belle-sœur, de sa chambre à coucher, elle voit les éoliennes. Nous, de notre salle de bain - et on a un sauna dans notre salle de bain, donc avec une vue sur la nature on voit l'éolienne ! » (Membre d'une association de riverains opposants.)
}

Parmi les autres acteurs du débat éolien, on a bien du mal à en trouver qui accordent une place de représentants de citoyens aux associations d'opposants. Les acteurs ne parviennent pas à considérer ces dernières autrement que comme des lobbies mobilisés pour la défense d'intérêts privés - ce qu'ils jugent incompatible avec leur pleine participation à un processus de construction commune. En outre, leurs discours sont considérés comme «bétonnés » et donc offrant peu de perspectives en vue de réels échanges. Enfin, les représentants de telles associations ont un comportement jugé violent et inadéquat avec un exercice démocratique - violences verbales, voire violences physiques latentes. Si les acteurs publics cherchent à affirmer que ces associations peuvent être une voix comme une autre dans un processus polyphonique, ils ne voient pas pour autant dans leurs adhérents une figure du citoyen.

\section{Le citoyen administré}

Une autre figure du citoyen est esquissée par les enquêtés : 1'administré, figure centrale de la démocratie représentative chère en particulier aux maires. On est ici à l'échelle de la commune : le citoyen reste là encore assez proche du riverain. Cette figure met en valeur celle de l'élu, considérée comme centrale dans le développement éolien. Pour les promoteurs, l'élu est un personnage-clé de la réussite du projet, bien qu'il n'ait aucun pouvoir décisionnel direct. Les opposants organisés aussi lui attribuent un rôle central. Ils mettent en avant son devoir de protection des citoyens et souhaitent que sa responsabilité soit engagée.

« L'intérêt de l'élu, c'est quand même de faire en sorte que le projet se passe bien, parce que c'est quand même le représentant des administrés. Donc... s'il veut pas avoir des administrés qui soient contre le projet, donc finalement qui pourraient arriver à être contre lui, il a tout intérêt à ce que ça se passe bien [...], [à] avoir un peu ce rôle de médiateur. » (Médiateur, BE en médiation environnementale.)

"Quand on est élu d'une municipalité [...] la première chose, c'est représenter ses administrés... Représenter l'intérêt général. Protégeant les concitoyens. [...] Le jour du conseil municipal, on a déposé en mains propres chez chaque conseiller municipal un courrier: "Écoutez, vous allez prendre une décision - bon, on a demandé au maire à faire un vote à main levée-, donc c'est vous qui allez engager notre avenir. Et nous vous demanderons des comptes. Vous personnellement. Si ça se passe mal." » (Responsable d'une association environnementaliste d'opposants.)

\section{L'habitant}

Enfin, une figure semble émerger autour du terme d'habitant. Elle est portée par une réinterprétation de la question du paysage par les acteurs du développement éolien, au premier rang desquels les acteurs publics et des acteurs privés impliqués dans les études liées aux projets, dont des paysagistes.

En effet, comme nous l'avons mentionné précédemment, ces acteurs font du thème du paysage une entrée pour élaborer des outils d'aide à la décision. Dans un premier temps ${ }^{21}$, ils mobilisent la catégorie de " paysages emblématiques »- qu'ils vont contribuer à renforcer significativement, au point qu'elle deviendra « emblématique » de la question éolienne. On y trouve des paysages réglementairement protégés ou faisant l'objet de représentations artistiques ou de valorisations touristiques - brochures, guides. La demande sociale de paysages est donc assimilée aux paysages référencés, presque institutionnalisés. Rapidement, quelques acteurs, dont les principaux « fondateurs » de la notion, vont en montrer les limites et insister sur la notion de paysages ordinaires qu'elle appelle.

Ils ont recours à un ensemble de termes significatifs de l'évolution de la problématique du paysage dans les débats éoliens: covisibilité, intervisibilités, zone

\footnotetext{
${ }^{21}$ Lors de l'élaboration de la charte départementale des éoliennes.
} 
d'influence visuelle... Ces termes énoncent qu'il faut changer d'échelle spatiale quand on parle d'éolien. Il y a un élargissement de la focale, depuis le site éolien jusqu'à la commune, puis à un territoire beaucoup plus vaste : un parc éolien est visible parfois de plusieurs communes à la ronde et, pour peu qu'il soit relayé visuellement par un autre parc, l'étendue à prendre en compte peut se révéler très conséquente. Ces termes sont porteurs d'un autre aspect, politique: celui qui concerne les interrelations entre personnes ou groupes sociaux, notamment dans ce qu'elles peuvent impliquer de tensions, voire de conflits. La figure pertinente est dès lors celle de l'habitant, celui qui se tient et se meut au quotidien dans les paysages « ordinaires » ou « banals ».

«On a établi la carte des paysages emblématiques. [...] Beaucoup étaient tentés de dire : "Ce qui est protégé, on n'y touche pas et ailleurs on fait n'importe quoi." [...] c'est une mauvaise politique que de raisonner comme ça. [...] C'est comme ça qu'on fabrique du désordre, $[\ldots]$ [qu'on] procède par exclusion. » (Responsable du service Prospective, DDE.)

«Y avait un paysagiste-conseil, moi, je voulais plus aller le voir [...]. En gros, dans l'approche du paysage que l'on avait, [...] [on prenait aussi en compte] le paysage perçu depuis les routes, depuis les habitants : en gros, le paysage de madame Michu quand elle va acheter le pain, quoi ! [...] Et toute cette partie-là du paysage perçu par le péquin moyen, [...] c'était le dernier des mépris et il le balayait. » (Paysagiste, BE.)

L'habitant serait celui qui peut légitimement, mais aussi sereinement, être convié dans les débats autour des projets éoliens : il se distingue donc radicalement du riverain véhiculant ses angoisses. En effet, le terme d'habitantn'est jamais associé, dans les entretiens, à l'idée d'une opposition viscérale motivée par la volonté de défendre des intérêts propres, une bulle intime. Pour certains enquêtés, au contraire, l'habitant, qui développe une relation existentielle à son environnement, serait susceptible d'établir des liens positifs forts avec cet objet qu'est l'éolienne. Plusieurs témoignages évoquent l'habitant comme l'individu qui «vit avec ses éoliennes », parfois personnifiées. Certains insistent sur le contact presque charnel qui peut s'établir avec cet objet particulier, animé quasiment au sens fort du terme. En bref, l'éolienne peut entrer dans la bulle intime des habitants.

« Donc les gens s'assoient, s'adossent, se posent adossés à... On peut la toucher, on peut sentir si ça vibre, on peut se mettre sous les pales, on peut enregistrer... On vit avec. [...] Quand elles sont arrêtées [....], les gens étaient tout malheureux. Ils appelaient : “Mais qu'est-ce qu'elles ont ? [...] elles sont malades ?" » (Secrétaire de mairie.)

«Y a aussi l'aspect paysage, où des gens [...] s'approprient les éoliennes, ils les connaissent, ils disent : "Celle-là, elle tourne plus que l'autre..." Parce qu'on les voit de loin, elles ont une vie et on voit... Les gens finissent par vivre avec leurs éoliennes, ils les connaissent... » (Porteur de projet.)

\section{L'habitant, entre droit à participer et droit à être laissé tranquille}

Les travaux évoqués ici montrent que les acteurs impliqués dans le développement éolien - qu'ils impulsent, accompagnent ce développement ou s'y opposent conçoivent le «public» principalement comme une masse informe - donc à informer ${ }^{22}$. Si ces acteurs appellent de leurs vœux la figure du citoyen, ils peinent à la reconnaître dans cette masse. Cette observation est cohérente avec nos analyses montrant que leurs conceptions pratiques de la participation sont peu à même de révéler cette figure (Le Floch et Fortin, à paraître). L' « absence » de celui qui est précisément fondéà participer aux affaires publiques dans un contexte démocratique peut paraître paradoxale à un moment où la participation est érigée au rang de principe démocratique incontournable. Qui donc, alors, se tiendrait derrière ce "public », cible des démarches dites participatives?

Les acteurs impliqués dans le développement éolien esquissent quelques figures. La principale est celle du riverain. Elle est éminemment problématique à leurs yeux. S'il est possible de négocier - notamment sur le mode marchand - avec le riverain propriétaire, ce n'est pas le cas avec le riverain hypersensible, qui considère l'installation d'éoliennes à proximité de chez lui comme une atteinte à l'intégrité de son patrimoine, de sa sphère intime, voire de sa personne. Les promoteurs du développement éolien comme les acteurs publics élaborant des outils d'aide à la décision tentent de contenir spatialement cette figure problématique, en insistant sur la question de la distance entre habitations et éoliennes. La dimension spatiale du riverain prend ainsi peu à peu de l'ampleur : 300, 500, 1000 mètres... Toutefois, face à l'évidente nécessité d'un changement d'échelle imposé en particulier par les caractéristiques physiques de l'éolienne, l'idée d'un seuil de distance perd rapidement de sa pertinence ; la figure à considérer change alors de nature.

$C^{\prime}$ est ainsi que le riverain cède la place à l'habitant. Ce dernier n'est plus celui pour qui l'éolienne est située à une certaine distance, mais celui pour qui l'éolienne est un élément de l'expérience des paysages ordinaires, quotidiens. Toutefois, les travaux relatés ici montrent que cette figure demeure mal définie. Ils rejoignent en cela des analyses faites par ailleurs (voir précédemment) ; il faut dire que, à l'exception de promoteurs ou de bureaux

\footnotetext{
${ }^{22}$ Assertion que les quelques entretiens supplémentaires conduits auprès d'habitants non impliqués dans les débats nuancent significativement: ces personnes ont une " conscience énergétique » certaine, qu'elles replacent bien souvent dans un contexte large tant spatialement - problèmes de l'indépendance énergétique de la Bretagne, du changement global que temporellement - militantisme antinucléaire au tournant des années 1980...
} 
d'études spécialisés dans le développement éolien, les acteurs entendus travaillent dans des domaines d'intervention divers. Deux lectures de cette figure mal définie sont possibles selon que l'on entend privilégier une approche critique - croisant la question des normes avec les discours sur les pratiques sociales, en focalisant sur la dimension empirique - ou une approche normative - focalisée sur les normes produites et/ou à promouvoir, selon une vision idéaliste.

Selon la première lecture, il y a un risque que la figure de l'habitant, peu explicitée et non stabilisée, demeure plus ou moins confondue avec celle du riverain, prégnante dans les débats éoliens. Le risque serait alors de déléguer le pouvoir aux mains de minorités de toutes sortes: personnes hypersensibles à tout changement vécu comme violation de l'intimité, fragiles sur le plan de la santé, fragiles psychologiquement... Cette tendance irait sans doute dans le sens d'un phénomène décrit par Mitchell (2005) : le développement d'un nouveau modèle de citoyenneté, fondé non plus sur l'idée d'actions collectives, mais sur le respect du droit à être laissé tranquille et son corollaire, le droit d'exclure ; un modèle qui serait en correspondance avec les voitures que nous conduisons, les « $\mathrm{SUV}^{23}$ », qui placent le conducteur comme maître de l'espace et le maintiennent à l'abri des intrus.

Selon la seconde lecture, l'habitant, conçu comme un être géographique - un être social et politique dont le rapport à la matérialité et à l'étendue terrestre fonde significativement les conditions d'existence-, semble une figure prometteuse en termes de participation. Irait-on vers une définition de celui qui participe qui ne soit plus seulement soit marchande (le riverain), soit sociologique et politique (le citoyen, l'administré...), mais qui intègre des considérations géographiques au sens plein du terme ? Une définition dans laquelle l'espace ne soit pas restreint à son sens de distance/proximité spatiale, mais soit pris également dans ses dimensions sociale et politique, voire sensibles: l'habitant est celui dont l'expérience de l'environnement compte. Une telle définition encouragerait la mutation du sujet réagissant (le riverain susceptible d'émerger de la masse du public pour s'opposer à un projet) en sujet agissant. Cet habitant serait un être humain géographique - un «être-au-monde» -, vivant les changements de son environnement - un environnement où local et global sont en interrelation -, voire y participant, et pas seulement en prenant part à des décisions ou des actions collectives via des procédures démocratiques. Plus généralement, il y participe ne serait-ce que parce qu'il partage une portion de surface terrestre avec d'autres,

\footnotetext{
23 Sports utility vehicle.
}

selon une conception qui rappelle l'origine étymologique commune des termes «participation » et « partage».

\section{Références}

Arnstein, S.R., 1969. A ladder of citizen participation, Journal of the American Institute of Planners, 35, 4, 216-224.

Berque, A., 2000. Écoumène: introduction à l'étude des milieux humains, Paris, Belin.

Beuret, J.-E., Dufourmantelle, N., Beltrando, V., 2006. L'Évaluation des processus de concertation: RELIEF, une démarche, des outils, Paris, La Documentation française.

Blondiaux, L., 2004. Démocratie délibérative et démocratie participative : une lecture critique, Conférences de la chaire $M C D$, Montréal, Chaire de recherche du Canada en mondialisation, citoyenneté et démocratie.

Blondiaux, L., Sintomer, Y., 2002. L'impératif délibératif, Politix, 15, 57, 17-35.

Bussi, M., 2001. Géographie, démocratie, participation: explication d'une distance, arguments pour un rapprochement, Géocarrefour, 76, 3, 265-272.

Carrel, M., 2007. Pauvreté, citoyenneté et participation. Quatre positions dans le débat sur la «participation des habitants", in Neveu, C. (Ed.), Cultures et pratiques participatives : une perspective comparée, Paris, L'Harmattan, 95-112.

Charles, L., Kalaora, B., 2003. Sociologie et environnement en France : l'environnement introuvable?, Écologie et politique, 27, 31-58.

Claeys-Mekdade, C., 2001. Qu'est-ce qu'une «population concernée » ? L'exemple camarguais, Géocarrefour , 76, 3, 217-223.

Corsin Jimenez, A., 2003. On space as a capacity, Journal of the Royal Anthropological Institute, 9, 137-153.

Fourniau, J.-M., 2008. La sélection des participants dans les dispositifs de démocratie participative: un citoyen plus amateur qu'ordinaire. Communication au colloque Sélection des acteurs et des instruments de l'action publique, SAIAP, Lyon, 26-28 juin.

Jobert, A., 1998. L'aménagement en politique, ou ce que le syndrome NIMBY nous dit de l'intérêt général, Politix, 11, $42,67-92$.

Le Floch, S., Fortin, M.-J., 2008. « Paysage », « co-visibilité » et esthétique autour de l'implantation d'éoliennes : vers une définition relationnelle de la qualité dans les projets de développement durable?, in Da Lage, A., et al. (Eds), L'Après-développement durable: espaces, nature, culture et qualité, Paris, Ellipses, 223-231.

Le Floch, S., Fortin, M.-J., à paraître. Les paysages d'Éole à l'épreuve du développement durable. Où accepter n'est pas toujours participer, in Luginbühl, Y., et al. (Eds), Paysages et développement durable, Versailles, Quæ.

Lussault, M., 2000. Action(s) !, in Lévy, J., Lussault, M. (Eds), Logiques de l'espace, esprit des lieux: géographies à Cerisy, Paris, Belin, 11-36. 
Massey, D., 1999. Space-time, "science" and the relationship between physical geography and human geography, Transactions (Institute of British Geographers), 24, 261-276.

Mermet, L., Berlan-Darqué, M. (Eds), 2009. Environnement: décider autrement. Nouvelles pratiques et nouveaux enjeux de la concertation, Paris, L'Harmattan.

Mitchell, D., 2005. The S.U.V. model of citizenship: Floating bubbles, buffer zones, and the rise of the "purely atomic" individual, Political Geography, 24, 77-100.

Reçu le 19 février 2010. Accepté le 3 février 2011.
Neveu, C., 2003. Citoyenneté et espace public : habitants, jeunes et citoyens dans une ville $d u$ Nord, Villeneuve-d'Ascq, Presses universitaires du Septentrion.

Painter, J., Philo, C., 1995. Spaces of citizenship: An introduction, Political Geography, 14, 2, 107-120.

Relph, E., 1976. Place and Placelessness, London, Pion. 